Revista Destaques Acadêmicos, Lajeado, v. 12, n. 3, 2020. ISSN 2176-3070

DOI: http://dx.doi.org/10.22410/issn.2176-3070.v12i3a2020.2703

http://www.univates.br/revistas

\title{
INFLUÊNCIA DA FISIOTERAPIA NA INDEPENDÊNCIA EM ATIVIDADES DE VIDA DIÁRIA E NO EQUILÍBRIO DE PACIENTES COM CONDIÇÕES CRÔNICAS ATENDIDOS NA CLÍNICA-ESCOLA DE FISIOTERAPIA DA UNIVATES
}

\author{
Laura da Silva Lopes ${ }^{1}$, Magali Teresinha Quevedo Grave ${ }^{2}$
}

Resumo: Introdução: As doenças crônicas não transmissíveis (DCNT) são um problema de saúde global e uma ameaça à saúde e ao desenvolvimento humano. A Organização Mundial da Saúde (OMS) estima em cerca de 36 milhões as mortes anuais causadas por esse grupo de doenças. No Brasil o quadro das DCNT é de grande magnitude, correspondendo a $72 \%$ da mortalidade total no país em 2017, apesar de ter-se verificado, nas últimas décadas, uma redução de cerca de $20 \%$ nas taxas de mortalidade por DCNT, particularmente pelo aumento da mortalidade por doenças cardiocirculatórias, vasculares e respiratórias crônicas. A fisioterapia tem um papel importante no tratamento de pessoas com DCNT, cujas funções motoras são afetadas. Objetivo: Verificar a influência do tratamento fisioterapêutico em pacientes com sequelas neuromotoras decorrentes de DCNT atendidos na Clínica-Escola de Fisioterapia (CEF) da Universidade do Vale do Taquari - Univates, Lajeado/RS. Metodologia: Estudo retrospectivo, descritivo e transversal, de análise quantitativa, de prontuários de usuários da $\mathrm{CEF}$, com idade igual ou superior a 18 anos, diagnóstico de alguma DCNT, que receberam atendimento de fisioterapia, no mínimo, uma vez por semana, cujo prontuário apresentasse ao menos uma avaliação e uma reavaliação através do Índice de Barthel (IB) e da Escala de Equilíbrio de Berg (EEB). Resultados: Foram analisados 194 prontuários, do quais 14 cumpriam os critérios de inclusão. A idade dos pacientes variou de 27 a 88 anos (média: 56,84; DP:20,88); nove (64\%) do sexo masculino, sete (50\%) com diagnóstico de acidente vascular cerebral, quatro (15\%) com doença de Parkinson, dois (29\%) com traumatismo crânio encefálico e um (7\%) com encefalopatia crônica não progressiva da infância. Com relação ao índice de Barthel, quatro pacientes mantiveram a pontuação máxima de 100 pontos nas reavaliações (R1 e R2), um paciente manteve a pontuação de 95 pontos em R1 e nove apresentaram

1 Graduanda em Fisioterapia. Universidade do Vale do Taquari - Univates, laura.lopes@ universo.univates.br

2 Doutora em Medicina e Ciências da Saúde pela PUCRS. Orientadora. Universidade do Vale do Taquari - Univates, mgrave@univates.br 
aumento na pontuação, quando comparados os resultados da avaliação. Os resultados da EEB se mantiveram para dois pacientes; os demais apresentaram ganhos numéricos quando comparados os valores da avaliação aos da R1 e R2. Conclusão: através dos protocolos do IB e EEB, foi possível constatar que a fisioterapia é efetiva no tratamento de pessoas com sequelas neurológicas decorrentes de DCNT.

Palavras-chave: Condições crônicas. Fisioterapia. Equilíbrio. Independência funcional.

\section{INTRODUÇÃO}

Indivíduos com doenças crônicas não transmissíveis (DCNT) apresentam limitações funcionais que interferem na sua qualidade de vida. Essas limitações podem variar, desde uma simples falta de ar, até uma dependência total de terceiros (FELÍCIO et al., 2005). São consequências das alterações causadas por DCNT de origem neural o desalinhamento postural, o déficit de força muscular, as alterações no equilíbrio e na flexibilidade, a amplitude de movimento, a marcha e a capacidade funcional em atividades de vida diária (AVD) (LIMA; PETRIBÚ, 2016). Ainda, podem ocorrer comprometimentos psíquicos, sociais e econômicos, que influenciam na condução da vida desses sujeitos (PIASSAROLI et al., 2012).

Dentre as DCNT de maior prevalência, que afetam a motricidade, função e qualidade de vida das pessoas, pode-se destacar o Acidente Vascular Encefálico (AVE), a Doença de Parkinson (DP), a Encefalopatia Crônica Não Progressiva da Infância (ECNPI) e o Traumatismo Crânio Encefálico (TCE), sendo esses os diagnósticos mais prevalentes nos pacientes que buscaram por atendimento fisioterapêutico no período do presente estudo.

O AVE é a principal causa de mortes por doenças cerebrovasculares no Brasil. São aproximadamente 16 milhões de pessoas acometidas por ano, com seis milhões de mortes pela doença, anualmente, no mundo. Em 2016, foram internadas, pelo Sistema Único de Saúde (SUS), um total de 188.223 pessoas para tratamento de AVE e, desse total, foram registradas 40.019 mortes. O AVE pode ser classificado como isquêmico ou hemorrágico; o primeiro ocorre devido à oclusão de artérias encefálicas, levando à diminuição de aporte sanguíneo ao tecido nervoso, enquanto o AVE hemorrágico acontece mediante ruptura de um ou mais vasos, ocasionando sangramento intracraniano. Pessoas que sofreram AVE podem apresentar mobilidade articular diminuída, alterações na linguagem e cognição, rigidez nos membros comprometidos, limitação na independência motora e déficits funcionais (DALPIAN; GRAVE; PÉRICO, 2013).

A Doença de Parkinson (DP) é uma doença de caráter degenerativo, caracterizada pela perda progressiva de neurônios da parte compacta da substância negra, situada no mesencéfalo. A degeneração nesses neurônios é irreversível e resulta na diminuição da produção de dopamina, que é um neurotransmissor essencial no controle dos movimentos, causando acinesia, rigidez, tremor e instabilidade postural. É uma moléstia crônica e progressiva 
do sistema nervoso central que acomete principalmente o sistema motor, além de manifestações não motoras como distúrbios do sistema nervoso autônomo e alterações do sono. A prevalência da DP na população é de 100 a 150 casos por 100.000 habitantes (BARBOSA; SALLEM, 2005).

Entende-se por encefalopatia crônica não progressiva da infância (ECNPI) um conjunto de alterações do movimento e da postura decorrente de uma lesão no encéfalo, ainda imaturo, nos períodos pré, peri ou pós-natal. A parte motora é comprometida, resultando na modificação do tônus muscular, no desalinhamento postural e em dificuldades para realizar movimentos contra a gravidade. Distúrbios associados, como deficiência mental, déficits visuais, sensoriais e de linguagem, podem ocorrer (GRAEFF; GRAVE; PÉRICO, 2011). Estudos epidemiológicos descrevem que, no Brasil, estima-se de 30.000 a 40.000 novos casos de ECNPI por ano (FERREIRA, 2012).

O traumatismo crânio encefálico (TCE) caracteriza-se por uma lesão neurológica, de origem traumática, que pode resultar no comprometimento das funções cognitivas, motoras e comportamentais, concomitantemente. As causas mais comuns do TCE são decorrentes de acidentes automobilísticos, quedas e acidentes por arma de fogo (GAUDÊNCIO; LEÃO, 2013). No Brasil, óbitos decorrentes de TCE apresentam taxa de mortalidade de cerca de 5 casos por 100 mil habitantes ao ano (MAGALHÃES et al.; 2017).

Considerando o crescimento expressivo das doenças neurológicas que afetam a independência e a qualidade de vida das pessoas, a necessidade de um acompanhamento fisioterapêutico tem sido cada vez maior, uma vez que estimula a reaprendizagem motora, incentiva a independência funcional e promove a autonomia de pessoas com DCNT decorrentes de lesões ao sistema nervoso. Nesse sentido, instrumentos de avaliação que permitam acompanhar a evolução desses pacientes quanto à eficácia do tratamento proposto também são importantes, na medida em que fornecem feedback para as condutas terapêuticas elencadas no plano de tratamento (HAASE; MACHADO; OLIVEIRA, 2008).

Dentre os protocolos considerados padrão ouro para avaliação da independência funcional em AVD e do equilíbrio, tem-se o Índice de Barthel (IB) e a Escala de Equilíbrio de Berg (EEB), respectivamente (MINOSSO et al., 2010; SOUZA; SANTOS, 2012).

O IB apresenta domínios que avaliam o quão independente é a pessoa em AVD, apresentando resultados quantitativos que se transformam em subsídios para o planejamento de estratégias no atendimento. É organizado em 10 itens: alimentação, banho, higiene pessoal, vestir-se, intestino, bexiga, transferência para higiene íntima, transferência cadeira e cama, deambulação e subir escadas.

A Escala de Equilíbrio de Berg (EEB) está fortemente ligada à avaliação do equilíbrio estático e dinâmico, buscando evidenciar o quão propenso o sujeito está ao risco de quedas. É formada por 14 itens: sentar-se, ficar de pé 
alcançar, girar, olhar acima dos ombros, subir e descer degraus e ficar em apoio unipodal (PIMENTEL; SCHEICHER, 2009).

Considerando o exposto, este estudo teve como objetivo verificar a influência do tratamento fisioterapêutico de pacientes com sequelas neuromotoras decorrentes de DCNT, atendidos na CEF da Univates, no que diz respeito à independência em AVD e equilíbrio, a partir da análise do IB e da EEB.

\section{METODOLOGIA}

Trata-se de um estudo retrospectivo, descritivo e transversal, análise quantitativa de dados secundários, realizado na Clínica-Escola de Fisioterapia (CEF) da Universidade do Vale do Taquari - Univates, Lajeado/RS. Para realizar a coleta de dados, inicialmente, obteve-se a autorização do Centro Clínico Univates (CCU) e, posteriormente, da CEF.

O público-alvo deste estudo caracteriza-se por usuários com distúrbios neuromotores decorrentes de DCNT, que realizaram atendimento fisioterapêutico apenas na CEF, no mínimo uma vez por semana, por pelo menos um semestre, no período de 2012 a 2019, com idade igual ou superior a 18 anos, cujo prontuário apresentasse pelo menos uma avaliação e uma reavaliação relacionadas à independência em AVD, através do IB e do equilíbrio, mensurado pela EEB.

Por se tratar de um estudo retrospectivo, de análise de dados secundários, conforme orientação do Conselho Nacional de Ética em Pesquisa (CONEP), o projeto não precisou passar por análise de um Comitê de Ética em Pesquisa (CEP), entretanto, todos os critérios éticos foram respeitados. Os nomes dos pacientes foram substituídos por números (P1, P2 etc.) não havendo identificação dos mesmos. Da mesma forma, conforme prevê a Resolução $466 / 2012$, o material será guardado pela pesquisadora por um período de cinco anos e, após, será incinerado. Este estudo segue os preceitos éticos de pesquisa com seres humanos e foi aceito pelo Centro Clínico e Clínica Escola de Fisioterapia da Univates.

A análise dos prontuários foi realizada em duas etapas: primeiramente, a pesquisadora fez a leitura de todos os prontuários, disponibilizados de forma física, resultando na análise de 122 prontuários. Posteriormente, foi feita a leitura dos prontuários eletrônicos, já que a partir de abril de 2016, o sistema que passou a ser utilizado pela área da saúde da referida Instituição é o tasy, que fornece ferramentas de acesso à informação em tempo real, resultando na análise de 72 prontuários digitalizados.

Por fim, a pesquisadora realizou um rastreamento nas informações relacionadas à identificação dos pacientes, anotando-se dados sociodemográficos como idade, sexo, diagnóstico, data da avaliação e das reavaliações, número de atendimentos por semana e resultados para IB e EEB presentes nas avaliações 
e reavaliações, conforme critérios previamente estabelecidos. Os dados foram tabulados em planilhas no Excel e os nomes dos pacientes foram substituídos por números à medida que os prontuários foram sendo lidos.

\section{RESULTADOS}

Foram analisados todos os prontuários, físicos e eletrônicos, de pacientes encaminhados à atendimento fisioterapêutico na CEF da Univates, no período de março de 2012 a março de 2019, que tinham como diagnóstico clínico DCNT decorrentes de insultos ao sistema nervoso. Assim, chegou-se a um total de 194 prontuários. Desses, apenas 14 continham o registro de pelo menos uma avaliação e uma reavaliação através dos protocolos do IB e da EEB, em, no mínimo, seis meses de atendimento fisioterapêutico ininterrupto, apenas na CEF.

Dos 180 protocolos que não contemplaram os critérios de inclusão, 50 decorriam de pacientes cadeirantes, 20 provinham de pacientes que foram avaliados pelo teste de Romberg (que identifica o equilíbrio estático); 20 compreendiam avaliação pelo teste timed up and go (que verifica a capacidade da pessoa de levantar e caminhar para frente, girar de voltar e sentar-se novamente); 26 tiveram a independência em AVD avaliados pelo protocolo da medida de independência funcional (MIF - que detecta a carga de cuidados demandada por uma pessoa para a realização de tarefas motoras e cognitivas de vida diária); 34 protocolos estavam incompletos e 30 prontuários registravam que os pacientes realizavam fisioterapia em outros espaços.

A Tabela 1 registra o perfil sociodemográfico da amostra, diagnóstico, tempo de atendimento (tendo-se como referências os meses de fevereiro, julho e dezembro de cada ano) e os resultados alcançados na avaliação e nas reavaliações (R1 e R2) do IB e da EEB. Foram incluídos na pesquisa os prontuários de 14 pacientes com idades entre 27 e 88 anos $(56,84 \pm 20,88)$, sendo as doenças de maior incidência AVE, com um total de 07 casos (50\%), seguida da DP, com 04 pacientes (29\%), TCE com 02 casos (15\%) e um registro de ECNPI (7\%). Identificaram-se nove pacientes do sexo masculino (64\%) e 05 do sexo feminino $(28,6 \%)$. Com exceção de P11, os demais receberam atendimento de fisioterapia, na CEF da Univates, duas vezes por semana, durante seis ou 12 meses. 
Tabela 1 - Dados sociodemográficos e tempo de atendimento, com os resultados do IB e EEB na avaliação e após intervenção fisioterapêutica.

\begin{tabular}{|c|c|c|c|c|c|c|c|c|c|c|c|c|c|}
\hline P & Sexo & $\begin{array}{c}\text { Idade } \\
\text { (anos) }\end{array}$ & D & $\begin{array}{c}\text { Temp. de } \\
\text { aten. Meses }\end{array}$ & Avaliação & IB & EEB & R 1 & IB & EEB & R 2 & IB & EEB \\
\hline P1 & F & 63 & DP & 12 & Fev/2012 & 100 & 46 & Jul/2012 & 100 & 50 & Dez/2013 & 100 & 52 \\
\hline P2 & M & 66 & AVE & 12 & Fev/2012 & 80 & 24 & Jul/2012 & 85 & 38 & Dez/2012 & 90 & 43 \\
\hline P3 & F & 60 & DP & 6 & Jul/2012 & 100 & 47 & Ju1/2012 & 100 & 50 & - & - & - \\
\hline P4 & M & 70 & AVE & 12 & Jul/2012 & 100 & 50 & Dez/2012 & 100 & 53 & Jul/2013 & 100 & 55 \\
\hline P5 & M & 67 & AVE & 6 & Jul/2012 & 55 & 40 & Dez/2012 & 75 & 52 & - & - & - \\
\hline P6 & M & 59 & AVE & 12 & Jul/2013 & 90 & 43 & Dez/2013 & 100 & 43 & Jul/2014 & 100 & 50 \\
\hline P7 & M & 47 & TCE & 6 & Jul/2013 & 45 & 41 & Dez/2013 & 95 & 52 & - & - & - \\
\hline P8 & F & 72 & AVE & 12 & Fev/2014 & 80 & 48 & Ju1/2014 & 95 & 53 & Dez/2014 & 95 & 53 \\
\hline P9 & F & 41 & ECNPI & 12 & Fev/2017 & 75 & 30 & Jul/2018 & 85 & 38 & Dez/2019 & 85 & 38 \\
\hline P10 & M & 75 & DP & 12 & Fev/2018 & 100 & 50 & Ju1/2019 & 100 & 50 & Dez/2019 & 100 & 50 \\
\hline P11 & M & 88 & AVE & 12 & Jul/2018 & 55 & 38 & Dez/2018 & 55 & 38 & Jul/2019 & 55 & 38 \\
\hline P12 & M & 60 & AVE & 12 & Fev/2019 & 85 & 40 & Ju1/2019 & 95 & 51 & Dez/2019 & 100 & 52 \\
\hline P13 & F & 27 & TCE & 6 & Fev/2019 & 40 & 21 & Jul/2019 & 55 & 40 & - & - & - \\
\hline P14 & M & 79 & DP & 6 & Jul/2019 & 95 & 50 & Dez/2019 & 95 & 52 & - & - & - \\
\hline
\end{tabular}

Fonte: da autora (2020).

Legenda: Doenças de Parkinson (DP), Acidente Vascular Encefálico (AVE), Encefalopatia Crônica Não Progressiva da Infância (EPCNPI), e Traumatismo Crânio Encefálico (TCE), Paciente (P), Diagnóstico (D), Escala de Equilíbrio de BERG (EEB), Índice de Barthel (IB), Reavaliação 1 (R1), Reavaliação 2 (R2).

\section{DISCUSSÃO}

A CEF da Univates é um serviço-escola, inaugurado em outubro de 2007, a fim de atender os requisitos necessários para a formação qualificada dos futuros fisioterapeutas. Em 2010, passou a disponibilizar atendimentos aos usuários da rede de serviços de saúde da região do Vale do Taquari, mediante convênio com o Sistema Único de Saúde (SUS), o que possibilitou a ampliação dos atendimentos em fisioterapia e hidroterapia à comunidade, bem como serviços de fisioterapia realizados por estudantes matriculados em componentes curriculares que preveem o desenvolvimento de habilidades do futuro profissional.

Além do SUS, possui convênio com o Consórcio Intermunicipal de Saúde do Vale do Taquari (CONSISA) e com o Consórcio Intermunicipal de Serviços do Rio Pardo (CISVALE). O encaminhamento de usuários ao serviço de fisioterapia é feito por intermédio da Secretaria Municipal de Saúde, mediante requisição médica emitida em consulta com o médico da Estratégia de Saúde da Família (ESF) ou Unidade Básica de Saúde (UBS) de referência. Os atendimentos são realizados mediante triagem fisioterapêutica, para definição da área de reabilitação a que o usuário se destina, sendo elas: ortopedia e traumatologia, neurologia adulto e infantil, cardiorrespiratória, dermatofuncional, uroginecológica e estimulação precoce. Após, os usuários 
são avaliados e passam a ser atendidos na reabilitação, individualmente, ou em grupos e oficinas interdisciplinares de prevenção de agravos, promoção ou de educação em saúde.

Entre 2012 e 2019 foram realizados em torno de 39.618 atendimentos, sendo a demanda por serviços de reabilitação neurológica predominante sobre as demais áreas de atuação, ficando em torno de 40 a 55\% do total de atendimentos realizados. Isso se deve, provavelmente, à alta incidência de doenças neurológicas que causam déficits motores e funcionais e, possivelmente, por ter se tornado referência regional em reabilitação neurofuncional. Vale ressaltar que o maior percentual de pacientes que buscam fisioterapia nesta área é o dos acometidos por AVE, perfazendo um total de $60 \%$.

Diante do objetivo do presente estudo, de verificar a influência do tratamento fisioterapêutico em pacientes com sequelas neuromotoras decorrentes de DCNT, no que diz respeito à independência em AVD e no equilíbrio, através dos protocolos do IB e da EEB, alguns aspetos relevantes serão apontados, conforme descrito a seguir.

A escolha por protocolos de avaliação padronizados e validados, de acordo com o perfil físico-funcional do usuário são importantes, pois, por se tratar de pessoas com condições crônicas, na grande maioria das vezes, a reabilitação é demorada e a tendência é de que esses pacientes permaneçam por um longo período de tempo em tratamento fisioterapêutico; por outro lado, por ser um serviço-escola, a cada semestre, os pacientes são atendidos por estudantes diferentes, fazendo-se necessário o acompanhamento dos resultados da avaliação e tratamento desses pacientes, no decorrer do processo. Ainda, as informações obtidas através de uma avaliação físico-funcional criteriosa podem se transformar em ferramentas potentes no planejamento e eficácia das intervenções.

Com relação à influência da fisioterapia na independência em AVD e equilíbrio de pacientes com distúrbios neuromotores, a partir dos resultados descritos na Tabela 1, fazem-se necessárias algumas considerações referentes aos protocolos utilizados, no contexto deste estudo.

O IB é um instrumento amplamente utilizado no Brasil e no mundo, para a avaliação da independência funcional e mobilidade em AVD. Um estudo de revisão sobre instrumentos de avaliação do estado funcional de indivíduos com déficits motores, realizado em 2018, identificou alto índice de confiabilidade interexaminadores e intra-examinadores (ICC-0,99), (ICC-0,99) (PAIXÂO JÚNIOR, REICHENHEIN, 2018).

Da mesma forma, a EEB é das escalas mais utilizadas na avaliação de pessoas com danos motores de pacientes que deambulam e avalia o equilíbrio do indivíduo em atividades como andar, transferir-se e ficar em pé; é preditiva de queda em grupos com alterações de equilíbrio e apresenta boa confiabilidade 
interexaminadores (ICC-0,98) e intraexaminadores (ICC-0,99) (SOARES et al., 2009).

A Tabela 1 descreve o perfil sociodemográfico dos pacientes e seus diagnósticos mostrando que sete apresentam AVE e, desses, seis eram do sexo masculino (P2, P4, P5, P11, P12), com mais de 60 anos, exceção de P6, que à época da avaliação estava com 59 anos. Sabe-se que o número de indivíduos com mais de 60 anos de idade vem crescendo acentuadamente nas últimas décadas em todo o mundo, com projeções indicando que, em 2050, esse grupo contará com cerca de 1.900 milhões de pessoas. No Brasil, essa faixa da população apresentou um crescimento de 33,65\% no período de 2010 a 2016, e, com isso, as DCNT passaram a ser predominantes, com destaque para as Doenças Cardiovasculares, dentre as quais destaca-se o AVE, uma das principais causas de morte e incapacidade no Brasil e no mundo (JORGE et al., 2008).

Estudo realizado por Souza-Uva et al. (2012), observou que a taxa de incidência de AVE tem-se mantido sempre superior nos homens, o que pode estar relacionado à maior prevalência de hipertensão arterial e consumo de cigarro nesse sexo, ratificando os dados encontrados neste estudo.

Os resultados da presente pesquisa demonstram, a partir do IB e da EEB, que os pacientes com diagnóstico de AVE - P2 (M; 66 anos), P5 (M; 67 anos), P6 (M; 59 anos), P8 (F; 72 anos) e P12 (M; 60 anos) - apresentaram ganhos numéricos na avaliação e reavaliações, a partir do tratamento fisioterapêutico. P2 obteve pontuações de 80/85/90 no IB e 24/38/43 na EEB; P4 manteve 100 pontos no IB e obteve 40/52 na EEB; P5 passou de 55 para 75 no IB e de 40 para 52 pontos na EEB; P6 obteve 90 pontos na avaliação do IB, evoluindo para 100 na R1, mantidos em R2; a pontuação de P8 foi 80/95/95 e 48/53/53, respectivamente no IB e EEB; P12 evoluiu de 85 para 95 e 100 pontos no IB e de 40/51/52 na EEB e, por fim, P11 (M; 88 anos) manteve as pontuações decorrentes da avaliação - 55 pontos no IB e 36 pontos na EEB - em R1 e R2.

Importante destacar, com relação ao $\mathrm{IB}$, que a pontuação varia de 0 a 100 pontos, sendo que um resultado de 0 a 15 indica que a pessoa apresenta dependência total de terceiros para a realização de AVD; de 20 a 35 pontos, a dependência é considerada grave; quando a pontuação varia de 40 a 55 pontos, considera-se uma dependência moderada; resultados entre 60 e 95 pontos indicam dependência leve e 100 pontos demonstram independência total em atividades do dia a dia (WALTER, GRAVE, PÈRICO, 2009).

Isso significa dizer, considerando os resultados de nossa amostra, que P2 e P8, mesmo tendo obtido ganhos numéricos em R1 e R2, mantiveram-se na faixa de 60 a 95 pontos do IB, indicando dependência leve em AVD; P4 já demonstrava independência total, permanecendo nesta faixa em R1 e R2; P5 passou da faixa de dependência moderada para leve; P6 passou da faixa numérica de dependência leve para independência total em R2; P11 mantevese na faixa da dependência moderada e P12 passou de dependência leve para independência total em R2. Assim, apenas P11 não obteve ganhos quantitativos 
em R1 e R2, o que pode estar relacionado à sua idade, sendo esse o paciente com a idade mais avançada do presente estudo.

A EEB atinge um máximo de 56 pontos; pontuações até 20 denotam grande prejuízo no equilíbrio, de 21 a 40 pontos o equilíbrio é considerado aceitável e de 41 a 56 pontos o equilíbrio é considerado como um bom equilíbrio. Quanto mais próximo de 56 pontos, menor é o risco de quedas. Dos pacientes com AVE, com exceção de P11, todos obtiveram ganhos numéricos na EEB; R1 e R2, entretanto, se mantiveram na faixa de 41 a 56 pontos, ou seja, um bom equilíbrio. Provavelmente esses ganhos numéricos tenham se traduzido em ganhos qualitativos. P11 manteve 38 pontos em R1 e R2.

Um estudo realizado por Scalzo et al. (2011), com 8 pacientes do sexo masculino, com média de idade de 72,3 anos, pós AVE, no qual foi realizado treinamento semanal com condutas voltadas especificamente para a melhora do equilíbrio dinâmico, constatou que seis dos participantes obtiveram melhora no escore da EEB, passando de uma pontuação média de 40 para 50 pontos, quando comparados os resultados iniciais e finais, após 6 meses de fisioterapia, duas vezes por semana.

Outro estudo realizado com seis pacientes hemiplégicos, com o objetivo de verificar o grau de alteração do equilíbrio estático, dinâmico e de comprometimento do tronco, após AVE, através da Escala de Deficiências de Tronco (EDT) e EEB, no qual os pacientes foram divididos em grupo intervenção (GI) e controle (GC), identificou que o GI, que realizou protocolo de atendimento fisioterapêutico específico com exercícios de alongamento, de equilíbrio e de fortalecimento dos músculos do tronco, obteve aumento na pontuação de ambas as escalas na avaliação final, após 10 sessões de fisioterapia. No GC, que permaneceu em atendimentos convencionais de fisioterapia solo, apenas um paciente aumentou o escore na EDT (FERLA; GRAVE; PERICO, 2015).

Em um estudo retrospectivo envolvendo coleta de dados em prontuários de 109 pacientes acometidos por AVE, sendo 43 (60,56\%) homens e $28(39,44 \%)$ mulheres, com faixa etária média de 64 anos, atendidos na clínica-escola de fisioterapia da Universidade Metodista de São Paulo (UMESP), no período de fevereiro de 2001 a junho de 2003, verificou, através da análise dos resultados do IB, em relação aos ganhos funcionais nas AVD, melhora significativa entre a avaliação inicial e final. No entanto, apenas $23,94 \%$ dos prontuários continham essa informação (RODRIGUES; SÁ; ALOUCHE, 2004). A falta de informações nos prontuários também foi um problema encontrado em nosso estudo.

Klotz, Lucareli e Carlik (2005) realizaram um estudo com 22 pacientes com sequelas crônicas de AVE, sendo 13 do sexo masculino e 9 do sexo feminino, com idade média de 63 anos, com sequela de AVE há mais de 6 meses, tratados na clínica de fisioterapia da Universidade Pompéia (UNIP/SP), com três sessões semanais de 50 minutos de fisioterapia clássica, durante 45 dias, com o objetivo de verificar a influência do tratamento fisioterapêutico nas AVD dos pacientes, por meio do IB, previamente ao tratamento. Observou-se, nesse 
estudo, que a análise estatística indicou diferença significante para a variável evacuação, enquanto nas demais variáveis foi registrado um aumento no resultado final, apesar de não significante estatisticamente. Para os autores, é, portanto, possível sugerir que a intervenção fisioterapêutica, quando realizada em pacientes crônicos, esbarra em vícios biomecânicos adquiridos ao longo da instalação dos déficits motores, o que pode dificultar a evolução clínica.

Ao traçar o perfil sociodemográfico e a funcionalidade de 16 pacientes com diagnóstico de AVE, atendidos na clínica de fisioterapia neurológica da Universidade de Passo Fundo (UPF), no período de março a agosto de 2006, após intervenção fisioterapêutica convencional, duas vezes por semana, durante 6 meses, o estudo de Polesi et al. (2008) concluiu que 50\% dos pacientes permaneceram na faixa de pontos considerados independentes; $30 \%$ passou de dependência moderada para leve e $20 \%$ se mantiveram na categoria de dependência grave, necessitando de assistência permanente para realização de atividades de vida diária (POLESI et al., 2008).

Outra enfermidade que causa déficit de equilíbrio e dependência em AVD é a DP, uma afecção crônica e progressiva do sistema nervoso, caracterizada pelos sinais cardinais de rigidez, acinesia, bradicinesia, tremor e instabilidade postural que se acentuam com o evoluir da doença. De acordo com Souza et al. (2010), estima-se uma população de cerca de 200 mil indivíduos com DP, no Brasil. A prevalência em pessoas com idade entre 60 e 69 anos é de 700/100.000, e entre 70 e 79 anos é de 1500/100.000. No entanto, 10\% dos doentes têm menos de 50 anos e $5 \%$ têm menos de 40 anos. Além disso, 36 mil novos casos surgem, por ano, no país.

No presente estudo, os pacientes com DP apresentam faixa etária entre 60 e 79 anos, sendo que P1 (F; 63 anos), P3 (F; 60 anos) e P10 (M; 75 anos) mantiveram pontuações de 100 pontos no IB, sendo considerados independentes em AVD; P14 (M; 79 anos) manteve grau de dependência leve, com pontuação de 95 pontos, antes e após intervenção fisioterapêutica.

Na avaliação do equilíbrio, conforme proposto na EEB, P1 passou de 46 para 50 e 52 pontos em R1 e R2; P3 foi de 47 para 50 pontos; P10 manteve os 50 pontos em R1 e R2 e P14 manteve a pontuação de 41 pontos. No entanto, todos se mantiveram na faixa de 41 a 50, o que é considerado um bom equilíbrio. Se faz importante ressaltar esses resultados, já que a DP é uma doença degenerativa que, com o passar dos anos, causa perda das funções motoras e cognitivas, com interferência no equilíbrio e na independência em AVD, ou seja, o tratamento fisioterapêutico retarda e minimiza seus efeitos deletérios (MASACRENHAS; SOUZA, 2010).

Um estudo realizado com dez pacientes com DP, sendo 8 homens e 2 mulheres, com idades entre 70 e 82 anos, que receberam atendimento de fisioterapia com uma série de atividades de alongamento, dança e circuitos, durante 6 meses, duas vezes por semana, verificou que pacientes do sexo feminino (68 e 74 anos) e os dois homens mais jovens entre os homens (70 e 72 ) 
apresentaram evolução satisfatória na EEB após as intervenções, enquanto os demais mantiveram a pontuação inicial (CHRISTOFOLETTI et al., 2010). Esses resultados podem estar associados ao fator idade e ao tempo de manifestação da doença, visto que a DP é uma doença neurodegenerativa.

Mascarenhas e Souza (2010), ao analisarem a influência da fisioterapia em 24 pacientes com DP, de ambos os sexos, e idades entre 65 e 79 anos, que receberam um atendimento convencional de fisioterapia e um atendimento semanal de hidroterapia através do método de Bad Ragaz, durante seis meses, constatou, após reavaliação do IB, que $16(66,7 \%)$ pacientes mantiveram pontuações na faixa de 40 a 55 pontos - dependência moderada - e oito $(33,3 \%)$ mantiveram a pontuação de 100 pontos - independência total. Destaca-se, nesse caso, que as intervenções fisioterapêuticas têm o potencial de retardar a evolução da patologia, garantindo-se, assim, por mais tempo, autonomia e qualidade de vida.

Outra patologia, considerada como a principal causa mundial de morbimortalidade e incapacidade funcional, com maior predominância nos indivíduos adultos jovens com idade inferior a 45 anos, do sexo masculino, é o TCE, definido como qualquer agressão traumática gerada por forças externas capazes de ocasionar lesão anatômica ou comprometimento funcional de estruturas do crânio ou do encéfalo que, ao comprometer áreas encefálicas responsáveis pela motricidade, causa danos importantes na independência e funcionalidade dessas pessoas (SANTANA; SILVA; RODRIGUES, 2008).

Todo ano, cerca de 1,5 milhões de pessoas morrem e centenas de milhões requerem tratamento emergencial e posterior reabilitação física, decorrentes do TCE (TOLEDO et al., 2008). Neste estudo, P7 (M; 47 anos) e P13 (F; 27 anos) apresentam diagnóstico de TCE, sendo que P7 evoluiu satisfatoriamente nas AVD, passando de 45 para 95 pontos, no IB, e de 41 para 52 pontos na EEB; P13 alcançou, no IB e na EEB, respectivamente, 40/55 e 40/40 na avaliação e em R1.

Outro estudo, realizado com três adultos jovens do sexo masculino, de 22, 26 e 30 anos, três vezes por semana, durante quatro meses, utilizando a realidade virtual (RV) como forma de maximizar o equilíbrio, observou que todos os pacientes adquiriram melhora importante no desempenho do equilíbrio e no desempenho das AVD, sendo que as pontuações da EEE passaram, respectivamente, de 20/25/30 para 30/40/41 após o uso da RV e os resultados do IB variaram de 60/70/75 para 70/80/100 (SANTOS et al., 2013).

Através de um estudo de caso único realizado com paciente com diagnóstico de TCE, sexo masculino, de 24 anos, que recebeu intervenção fisioterapêutica em março e junho de 2016, duas vezes na semana, com duração de 50 minutos, através de exercícios realizados em solo e na plataforma vibratória, identificou-se ganhos numéricos de 20 pontos na EEB e de 25 pontos no IB, com melhora na capacidade funcional e diminuição do risco de quedas (PINHEIRO et al., 2017). 
No presente estudo, o único paciente com diagnóstico de ECNPI, P9, sexo feminino, de 41 anos, manteve suas pontuações nos protocolos do IB e EBB, respectivamente em 100/100/100 e 50/50/50, manifestando independência em AVD e no equilíbrio estático, dinâmico e marcha. As encefalopatias crônicas não progressivas da infância apresentam um espectro bastante diferente em termos de danos motores, cognitivos e de linguagem, podendo variar de leves a severos.

O estudo desenvolvido por Silva et al. (2015), envolvendo uma paciente com ECNPI, de 48 anos, durante 12 meses, uma vez por semana, mediante exercícios que incluíam o uso de cama elástica, bicicleta ergométrica e caminhada na esteira, constatou melhora significativa no equilíbrio dinâmico, passando de uma pontuação de 41 para 53 pontos na EEB.

$\mathrm{Na}$ perspectiva de analisar a funcionalidade de 10 indivíduos com ECNPI, de ambos os sexos, com idades entre 18 e 32 anos, classificados como hemiparéticos leves e moderados, através de caminhadas matinais associadas a alongamentos e exercícios respiratórios, três vezes por semana, durante três meses, o estudo de Flôr, Cardoso e Soldi (2008) constatou ganho funcional em AVD para $80 \%$ dos participantes, avaliados através do IB.

O AVE, a DP, o TCE e as ECNPI são doenças de alta incidência no Brasil e no mundo e estão relacionadas à perda das funções motoras e cognitivas, de forma lenta e progressiva, como no caso da DP e das ECNPI, ou imediatamente, assim como ocorre nos TCE e nos AVE, sejam eles isquêmicos ou hemorrágicos. As sequelas destas DCNT, na grande maioria dos casos, afetam a independência e a qualidade de vida destas pessoas, que passam a necessitar de um longo período de reabilitação.

\section{CONCLUSÃO}

Considerando o pequeno número de prontuários que continham os dados necessários para a análise proposta neste estudo, os dados aqui apresentados não podem ser generalizados, embora demonstrem que a fisioterapia, na grande maioria das vezes, melhora o desempenho em atividades de vida diária e no equilíbrio de pacientes com alterações motoras e funcionais decorrentes de DCNP, que afetam o sistema nervoso.

Outra conclusão importante, a partir deste estudo, é a necessidade de se ter padrões mínimos de avaliação de pacientes com distúrbios neuromotores, a partir da aplicação sistemática de protocolos pré-determinados, principalmente em se tratando de um serviço-escola, cuja quase totalidade de atendimentos é realizada por estudantes matriculados em componentes curriculares com carga horária prática e por estagiários em final de curso, fazendo com que, a cada semestre letivo, novos estudantes fiquem responsáveis pela avaliação e tratamento dos pacientes. 
O registro documental da aquisição de habilidades motoras e funcionais e da ocorrência de déficits ao longo de um período de tempo é fundamental para o prognóstico físico-funcional dos pacientes com DCNT que demandam de reabilitação física. A partir deste estudo, sugere-se a realização de análises mais aprofundadas, com relação à influência da fisioterapia na melhoria da qualidade de vidas desses sujeitos, mediante amostra mais robusta e análise de outros instrumentos de avaliação.

\section{REFERÊNCIAS}

BARBOSA, E. R.; SALLEM, F. A. S. Doença de Parkinson (diagnóstico). Revista Neurociências, v. 13, n. 3, p. 158-165, 2005. Disponível em: https:/ / periodicos.unifesp. br/index.php/neurociencias/article/view/8827. Acesso em: 24 jun. 2020.

CHRISTOFOLETTI, G. et al. Eficácia de tratamento fisioterapêutico no equilíbrio estático e dinâmico de pacientes com doença de Parkinson. Fisioterapia e Pesquisa, São Paulo, v. 17, n. 3, p. 259-263, 2010. Disponível em: https:/ /www.scielo.br/pdf/ fp/v17n3/13.pdf. Acesso em 15 jul. 2020.

DALPIAN, A. P. C.; GRAVE, M. T. Q.; PÉRICO, E. Avaliação da Percepção Corporal em Pacientes Pós-Acidente Vascular Cerebral (AVC). Revista Neurociências, v. 21, n. 3, p. 377-382, 2013. Disponível em: https:/ / periodicos.unifesp.br/index.php/ neurociencias/article/view/8162. Acesso em: 24 jun. 2020.

FELÍCIO, D. N. L. et al. Atuação do fisioterapeuta no atendimento domiciliar de pacientes neurológicos: a efetividade sob a visão do cuidador. Revista Brasileira em Promoção da Saúde, Fortaleza, v. 18, n. 2, p. 64-69, 2005. Disponível em: https:// www.redalyc.org/pdf/408/40818203.pdf. Acesso em 26 mai. 2020.

FERLA, F. L.; GRAVE, M.; PERICO, E. Fisioterapia no tratamento do controle de tronco e equilíbrio de pacientes pós AVC. Revista Neurociências, v. 23, n. 2, p. 211217, 2015. Disponível em: https:/ / periodicos.unifesp.br/index.php/neurociencias/ article/view/8028. Acesso em: 14 jul. 2020.

FERREIRA, H. C. Características do Sistema Respiratório na Encefalopatia Crônica não Progressiva da Infância. Revista Neurociências, v. 20, n. 1, p. 101-108, 2012. Disponível em: https://periodicos.unifesp.br/index.php/neurociencias/article/ view/8307. Acesso em: 24 jun. 2020.

FLÔR, J. da S.; CARDOSO, M; SOLDI, F. Medida da funcionalidade em adultos com paralisia cerebral. 2008. 58 f. Trabalho de Conclusão de Curso (Graduação) - Curso de Fisioterapia, Universidade do Sul de Santa Catarina, Tubarão. Disponível em: http:/ / fisio-tb.unisul.br/Tccs/08b/juliane_morgana/TCC.pdf. Acesso em: 17 jul. 2020.

GAUDÊNCIO, T. G.; LEÃO, G. de M. A Epidemiologia do Traumatismo CrânioEncefálico: Um Levantamento Bibliográfico no Brasil. Revista Neurociências, v. 21, n. 3, p. 427-434, 2013. Disponível em: https:/ / periodicos.unifesp.br/index.php/ neurociencias/article/view/8261. Acesso em: 24 jun. 2020. 
GRAEFF, D. R.; GRAVE, M. T. Q.; PÉRICO, P. Alterações neuromotoras e comprometimentos corporais encontrados em pacientes com Paralisia Cerebral em sete Instituições Especializadas do Vale do Taquari. Revista Destaques Acadêmicos, Lajeado, v. 3, n. 3, p. 13-20, 2011. Disponível em: http:/ / www.univates.br/revistas / index.php/destaques/article/view/108. Acesso em: 21 jun. 2020.

HAASE, D. C. B. V.; MACHADO, D. C.; OLIVEIRA, J. G. D. de. Atuação da fisioterapia no paciente com Doença de Parkinson. Fisioterapia em Movimento, v. 21, n. 1, p. 79-85, 2008. Disponível em: https:/ / periodicos.pucpr.br/index.php/fisio/ article/view/19033/18381. Acesso em: 26 mai. 2020.

JORGE, M. H. P. de M. et al. A mortalidade de idosos no Brasil: a questão das causas mal definidas. Epidemiologia e Serviços de Saúde, v. 17, n. 4, p. 271-281, 2008. Disponível em: http:/ / scielo.iec.gov.br/scielo.php?script=sci_ arttext\&pid=S1679-49742008000400004. Acesso em: 13 jul. 2020.

KLOTZ, T.; LUCARELI, P. R. G.; CARLIK, J. Avaliação das atividades de vida diária pelo Índice de Barthel de pacientes acometidos de acidente vascular encefálico. Fisioterapia Brasil, v. 6, n. 2, p. 108-111, 2005. Disponível em: http:/ / portalatlanticaeditora.com.br/index.php/fisioterapiabrasil/article/view/1971. Acesso em: 15 jul. 2020.

LIMA, A. de G. T.; PETRIBÚ, K. Acidente vascular encefálico: Revisão sistemática sobre qualidade de vida e sobrecarga de cuidadores. Revista Brasileira de Neurologia e Psiquiatria, v. 20, n. 3, p. 253-266, 2016. Disponível em: https: / /www.revneuropsiq. com.br/rbnp/article/view/168/107. Acesso em: 26 mai. 2020.

MAGALHÃES, A. L. G. et al. Epidemiologia do Traumatismo Cranioencefálico no Brasil. Revista Brasileira de Neurologia, v. 53, n. 3, p. 15-22, 2017. Disponível em: https://revistas.ufrj.br/index.php/rbn/article/view/12305. Acesso em: 28 jun. 2020.

MASCARENHAS, C. H. M.; SOUZA, M. P. Avaliação funcional de indivíduos portadores da doença de Parkinson, Arquivos de Ciência e Saúde, São José do Rio Preto, v. 17, n. 4, p. 179-184, 2010. Disponível em: http:/ / repositorio-racs.famerp.br/ racs_ol/vol-17-4/IDR\%203.pdf. Acesso em: 15 jul. 2020.

MINOSSO, J. S. M. et al. Validação, no Brasil, do Índice de Barthel em idosos atendidos em ambulatórios. Acta paulista de enfermagem, São Paulo, v. 23, n. 2, p. 218-223, 2010. Disponível em: https:/ / www.scielo.br/pdf/ape/v23n2/11.pdf. Acesso em: 26 mai. 2020.

PIASSAROLI, C. A. P. et al. Modelos de Reabilitação Fisioterápica em Pacientes Adultos com Sequelas de AVC Isquêmico. Revista Neurociências, v. 20, n. 1, p. 128137, 2012. Disponível em: https:/ / periodicos.unifesp.br/index.php/neurociencias / article/view/10341. Acesso em: 26 mai. 2020.

PIMENTEL, R. M.; SCHEICHER, M. E. Comparação do risco de queda em idosos sedentários e ativos por meio da escala de equilíbrio de Berg. Fisioterapia e Pesquisa, 
São Paulo, v 16, n. 1, p. 6-10, 2009. Disponível em: https:/ /www.scielo.br/pdf/fp/ v16n1/02.pdf. Acesso em: 24 jun. 2020.

PINHEIRO, A. I. T. et al. Abordagem fisioterapêutica em um paciente com traumatismo crânio encefálico (TCE): Estudo de caso. Encontro de Extensão, Docência e Iniciação Científica (EEDIC), v. 3, n. 1, 2017. Disponível em: http:/ / publicacoesacademicas.unicatolicaquixada.edu.br/index.php/eedic/article/ view/857. Acesso em: 17 jul. 2020.

RODRIGUES, J. E.; SÁ, M. da S. da S.; ALOUCHE, S. R. Perfil dos pacientes acometidos por AVE tratados na clínica escola de fisioterapia da UMESP. Revista Neurociências, v. 12, n. 3, p. 117-122, 2004. Disponível em: https:/ / periodicos.unifesp. br/index.php/neurociencias/article/view/8857. Acesso em: 16 jul. 2020.

SANTANA, W. R. de; SILVA, F. L. C.; Intervenção fisioterapêutica no atendimento em domicílio de pacientes com traumatismo crânio encefálico: Revisão integrativa. Revista Uningá, v. 55, n. 4, p. 7-16, 2018. Disponível em: <http:/ / revista.uninga.br/ index.php/uninga/article/view/2314>. Acesso em: 17 jul. 2020.

SANTOS, S. M. T. dos et al. Equilíbrio em Pacientes com Traumatismos Encefálicos que Praticam Natação e Realidade Virtual. Revista Neurociências, v. 21, n. 1, p. 89-93, 2013. Disponível em: https://periodicos.unifesp.br/index.php/neurociencias/article/ view /8215. Acesso em: 15 jul. 2020.

SCALZO, P. L. et al. Efeito de um treinamento específico de equilíbrio em hemiplégicos crônicos. Revista Neurociências, v. 19, n. 1, p. 90-97, 2011. Disponível em: https:/ / periodicos.unifesp.br/index.php/neurociencias/article/view/8416. Acesso em: 14 jul. 2020.

SILVA, F. P. da et al. Reeducação postural global em um adulto com paralisia cerebral: um estudo de caso. Fisioterapia e pesquisa, São Paulo, v. 22, n. 1, p. 90-96, 2015. Disponível em: https: / /www.scielo.br/pdf/fp/v22n1/1809-2950-fp-22-01-00090.pdf. Acesso em: 15 jul. 2020.

SOARES, K. V. et al. Avaliação quanto à utilização e confiabilidade de instrumentos de medida do equilíbrio corporal em idosos. Revista PubliCa, v. 1, n. 2, p. 78-85, 2009. Disponível em: https://periodicos.ufrn.br/publica/article/view/122. Acesso em: 24 jun. 2020.

SOUZA, A. C. da S.; SANTOS, G. M. Sensibilidade da Escala de Equilíbrio de Berg em indivíduos com osteoartrite. Motriz, Rio Claro, v. 18, n. 2, p. 307-318, 2012. Disponível em: https://www.scielo.br/pdf/motriz/v18n2/v18n2a11.pdf. Acesso em: 26 mai. 2020.

SOUZA, C. F. M. et al. A Doença de Parkinson e o Processo de Envelhecimento Motor. Revista Neurociências, v. 19, n. 4, p. 718-723, 2011. Disponível em: https:/ / periodicos. unifesp.br/index.php/neurociencias/article/view/8330. Acesso em: 13 jul 2020. 
TOLEDO, C. et al. Efeitos da fisioterapia respiratória na pressão intracraniana e pressão de perfusão cerebral no traumatismo cranioencefálico grave. Revista Brasileira de Terapia Intensiva, v. 20, n. 4, p. 339-343, 2008. Disponível em: https:/ / www.scielo.br/pdf/rbti/v20n4/v20n4a04.pdf. Acesso em 16 jul. 2020.

WALTER, J.; GRAVE, M. T. Q.; PÉRICO, E. Avaliação das habilidades psicomotoras e da motricidade global em paciente portadora da Doença de Huntington. Conscientiae Saúde, v. 8, n. 4, p. 655-663, 2009, Disponível em: https:/ / periodicos.uninove.br/ index.php?journal=saude\&page $=$ article $\&$ op $=$ view $\&$ path $\% 5 B \% 5 D=1980$. Acesso em: 24 jun. 2020. 